\title{
What is the best method to calculate the solar wind propagation delay?
}

\author{
B. Mailyan ${ }^{1}$, C. Munteanu ${ }^{2}$, and S. Haaland ${ }^{3, *}$ \\ ${ }^{1}$ Yerevan State University, Armenia \\ ${ }^{2}$ Alexandru Ioan Cuza, University Iasi, Romania \\ ${ }^{3}$ University of Bergen, Norway \\ *also at: Max-Planck Institute for Solar Systems, Germany
}

Received: 2 April 2008 - Revised: 29 May 2008 - Accepted: 2 June 2008 - Published: 5 August 2008

\begin{abstract}
We present a statistical study of propagation times of solar wind discontinuities between Advanced Composition Explorer (ACE) spacecraft orbiting the L1 libration point and the Cluster quartet of spacecraft near the Earth's magnetopause. The propagation times for almost 200 events are compared with the predicted times from four different models. The simplest model assumes a constant convective motion of solar wind disturbances along the Sun-Earth line, whereas more sophisticated models take the orientation of the discontinuity as well as the real positions of the solar wind monitor and target into account. The results show that taking orientation and real position of the solar wind monitor and target into account gives a more precise time delay estimation in most cases. In particular, we show that recent modifications to the minimum variance technique can improve the estimation of propagation times of solar wind discontinuities.
\end{abstract}

Keywords. Interplanetary physics (Discontinuities; Interplanetary magnetic fields; Instruments and techniques)

\section{Introduction}

Disturbances in Earth's magnetosphere, like e.g. aurora, magnetospheric storms and substorms, are often associated with disturbances in the solar wind, in particular directional changes in the interplanetary magnetic field (IMF). At the Earth's dayside magnetopause, a southward directed IMF can reconnect with the geomagnetic field, and allow energy and momentum to be transferred from the solar wind into the magnetosphere, and set up a large scale circulation of plasma in the magnetosphere (Dungey, 1961). It has also been argued that sudden northward turnings of the IMF can alter the magnetospheric equilibrium, and act as a trigger for mag-

Correspondence to: B. Mailyan

(bagrat_mailyan@yahoo.co.uk) netospheric substorms (Sergeev et al., 1986; Lyons, 1996; Lyons et al., 2003). The study of such interactions requires an exact timing of the IMF change at the Earth's dayside magnetopause.

A challenge in this connection is that solar wind measurements are usually taken at large distances away from Earth, for example by the Advanced Composition Explorer (ACE) spacecraft, and has to be time shifted in order to be representative for the Earth's upstream magnetopause. For ACE, the typical time shift to the Earth is of the order of one hour, depending on the solar wind speed. Although many studies have used one hour as a rule of thumb, a more careful approach requires observations from the solar wind, and then time shift the observations accordingly to be representative for the conditions at the frontside magnetopause.

Earlier studies, e.g. Ridley (2000); Horbury et al. (2001a,b); Weimer et al. (2002, 2003); Weimer and King (2008) have emphasized that not only the solar wind speed, but also the orientation of the IMF plays an important role for the propagation delay.

Horbury et al. (2001a) used data from the ACE spacecraft orbiting the L1 libration point and the Wind spacecraft closer to the Earth to study the propagation times of IMF discontinuities. Although they only considered IMF discontinuities with distinct southward turnings, they found that the best estimates of the propagation times were obtained if the orientation for each discontinuity was calculated from the cross product of the upstream and downstream magnetic field.

Weimer et al. (2003); Weimer and King (2008) took a different approach. They emphasized that knowledge about the IMF direction is important for any time intervals - not only during intervals with distinct discontinuities. In particular, the concurrent IMF direction (and partly also the time history of the solar wind) upstream of the Earth's magnetopause is used to parameterize magnetic field models (Tsyganenko, 2002a,b) and simulation models (e.g. Ogino et al., 1994; Gombosi et al., 2000). Likewise, statistical studies

Published by Copernicus Publications on behalf of the European Geosciences Union. 
of magnetospheric convection (e.g. Papitashvili and Rich, 2002; Weimer, 2005; Ruohoniemi and Greenwald, 2005, and references therein) sort the results according to concurrent IMF direction. Weimer et al. (2003) applied a running minimum variance analysis technique to determine the orientation of the IMF for each data point in a continuous time series of magnetic field data. The obtained normal, together with the solar wind velocity and spacecraft position is then used to predict the time delay between a monitor and a target for each data point. A large data set shifted according to this procedure (although with some modifications - see Sect. 3.2.3 below) is now available in electronic form through NASA's OMNIWEB system (http://omniweb.gsfc.nasa.gov/ html/ow_data.html). To our knowledge, the first large scale usage of this method to time shift IMF data were the convection studies by Haaland et al. (2007) and Förster et al. (2007).

More recently, Tsurutani et al. (2005) used measurements from ACE and Cluster to study the evolution of 7 distinct interplanetary magnetic field decreases and discontinuities preceeded by long periods of strong alfvénic wave activity. 6 of the 7 events were observed at both ACE and Cluster so that the propagation time could be established. Although they primarily focused on the steepening of the wave front during the propagation from ACE to Cluster, they also noted that the structures were essentially convected with the solar wind speed. The difference between the observed popagation time and the time predicted from a convective motion were less than a minute for all 6 cases.

In the present work, we have used data from Cluster mission and the ACE solar wind monitor to study the propagation delay of almost 200 well identified solar wind discontinuities. In particular, we test out the models of Weimer et al. (2003); Weimer and King (2008) on these events.

The paper is organized as follows; In Sect. 2, we give a brief overview of the data sources used in this study. Section 3 contains an overview of the procedures for calculating the time shift, as well as a brief description of the methods used to find the boundary normals of solar wind discontinuities. In Sect. 4, we show a case study and present the statistical results of the study. Section 5 summarizes the paper.

\section{Data sources}

Our primary data sources for this study have been the Advanced Composition Explorer (ACE) spacecraft in the solar wind and the Cluster spacecraft quartet near the Earth's magnetopause.

ACE was put into an approximately $40 \times 40 R_{E}$ Lissajous orbit around the L1 libration point some $1.5 \times 10^{6} \mathrm{~km}$ upstream of the Earth in 1998, and have since been frequently used as a solar wind monitor. The expected lifetime of ACE is until 2022, so ACE is going to be an important monitor of the solar wind also the next decade. In this work, we have used IMF data obtained from the ACE magnetic field instrument (MAG - see Smith et al., 1998), at 16-s resolution and plasma data from the ACE solar wind instrument (SWEPAM - see McComas et al., 1998). at 64-s resolution. These data sets were downloaded via the Coordinated Data Analysis Web (CDAWeb) facility (http://cdaweb.gsfc. nasa.gov/about.html).

To check the IMF near the Earth's upstream magnetopause, we have utilized observations from the Cluster satellites. Cluster is a European Space Agency (ESA) project comprising four identical satellites flying in close formation around the Earth. Cluster has a nearly $90^{\circ}$ inclination elliptical polar orbit, with perigee at around $4 R_{E}$ and apogee around $20 R_{E}$ geocentric distance, and an orbital period of approximately $57 \mathrm{~h}$. This orbit takes Cluster into the upstream solar wind during apogee in the months January to March every year. Our study is therefore focused on this season. All Cluster measurements are obtained within an $Y_{\mathrm{GSE}}$ range between -8 to $+8 R_{E}$, and $X_{\mathrm{GSE}}$ positions between approximately $14 R_{E}$ and Cluster's apogee around $20 R_{E}$.

Since we focus on IMF discontinuities, we have primarily used measurement from the magnetic field Experiment (FGM - see Balogh et al., 2001), but also data from the Cluster Ion Spectrometry (CIS) Experiment (Rème et al., 2001) were inspected to verify that Cluster was located in the solar wind. The Cluster data used are the official prime parameters with approximately 4-s time resolution, provided by the Cluster Data Center System (CSDS).

Our motivation was primarily to study the solar wind propagation delay, and not the evolution or properties of the solar wind discontinuities as such. We have therefore not utilized Clusters four-spacecraft capability in this study, and most of the Cluster measurements are taken from the $\mathrm{C} 3$ spacecraft. However, some of the events included in this work has also been studied by Knetter et al. (2004) and Knetter (2004), where the nature of the discontinuity as well as a comparison of single- and multi spacecraft methods are discussed in detail. In particular, these authors concluded that results based on four-spacecraft triangulation agreed fairly well with the cross product method, which we have also used.

\section{Methodology}

To calculate the exact propagation time of a plasma structure one needs to be able to uniquely identify the same structure at two locations in space. In practice, a reliable identification of a structure is only possible if the obeservations exhibit a distict signature which does not change much between the two locations. Interplanetary discontinuities, characterized by sharp changes in the direction or magnitude of the magnetic field are particularly suitable for this purpose. Since the beginning of space age, discontinuities have been extensively studied both from a theoretical view (e.g. Landau and Lifshitz, 1960; Hudson, 1970), but also experimentally (e.g. 
Colburn and Sonett, 1966; Siscoe et al., 1968; Turner and Siscoe, 1971; Smith, 1973).

The actual definition of the term discontinuity depends both on the measurements used (plasma, magnetic field or a combination of these) as well as the required change of that parameter. In the following we use the term discontinuity to describe events where we observe pronounced changes in the magnetic field direction within a time interval of less than a minute. As our intention is to study the propagation time, we did not specifically check whether these distinct changes matched the criteria used by, e.g. Tsurutani and Smith (1979) or Lepping and Behannon (1986) to identify discontinuities.

We first identified a large number of time segments containing distinct magnetic field rotations in the Cluster measurements when spacecraft quartet was located in the upstream solar wind. A number of these events could also be identified in the ACE magnetic field measurements. The events were initially selected by visually examining Cluster quick look plots (available at http://www.cluster.rl.ac.uk/ csdsweb-cgi/csdsweb_pick). To avoid discontinuities associated with magnetopause crossings or bow-shock activity, we also checked the Cluster nominal position and the CIS ion spectrogram. Only periods with Cluster positions on the dayside, outside the bow shock location which displayed ion temperatures around $1 \mathrm{keV}$ were considered. Cases where the same discontinuity could be observed at both ACE and Cluster were recorded and examined in more detail; The observed time shift between the ACE and Cluster observations were noted and compared to the time shift predicted by various models.

During the years 2001-2007, a total of 198 events with unambiguously matched signatures observed at both ACE and Cluster could be identified from this visual inspection of the data. We should emphasize that these 198 events by no means constitute the complete set of discontinuities for this period, but is a sufficient subset for our study. At this stage, we did not filter out any events, even if we suspected that one or more of the methods would fail or give poor results.

For time delay calculations, we tested four different methods, hereafter referred to as flat delay, cross product, minimum variance and constrained minimum variance. In the following, we give a brief description of each method.

\subsection{Flat delay}

This is the simplest way to estimate the solar wind propagation time between a monitor and a target near the upstream magnetopause. It assumes that a plasma element and the embedded IMF is convected at a constant speed along the SunEarth line (i.e. antiparallel to the $\mathrm{X}_{\mathrm{GSE}}$ axis) to the Earth's magnetopause. This approach, illustrated in the top panel of Fig. 1, is purely one-dimensional, and does not take into account either orientation of the IMF nor any displacement of the solar wind monitor away from the Sun-Earth line. The estimated time delay, $t_{\text {flat }}$, is then given by

$t_{\text {flat }}=\frac{\Delta x}{V x}$,

where $\Delta x$ is the distance between the solar wind monitor and the target along the Sun-Earth line, and $V x$ is the measured solar wind speed. As the solar wind propagation is predominantly along the $X_{\mathrm{GSE}}$ direction, the approximation $|V x| \simeq|V|$ is often used.

Since the method relies on the solar wind velocity only, it can in theory be applied to any time interval where the measurements of the velocity is available - not only time intervals containing distinct IMF discontinuities.

\subsection{Taking IMF direction into account}

As pointed out in e.g. Weimer et al. (2002, 2003), variations in the IMF are often contained in planar structures which are tilted at arbitrary angles with respect to the Sun-Earth line. (Weimer et al., 2003, refers to these tilted planar structures as IMF phase planes). As illustrated the lower panel of Fig. 1, a solar wind monitor displaced from the Sun-Earth line will measure the IMF at a different time (later or earlier, depending on the tilt direction of the phase plane) than it would if it had been located on the Sun-Earth line.

A more realistic calculation of the propagation delay would therefore have to take into account the orientation of these IMF phase planes as well as the real position of the solar wind monitor and target. Also, since the solar wind flow direction can have a significant $\mathrm{Y}$ or $\mathrm{Z}$ component, the full solar wind velocity vector should be used. Noting that the orientation of a planar structure or discontinuity can be described by its boundary normal, $\boldsymbol{n}$, the time delay can be expressed as:

$t_{d}=\frac{\left(\boldsymbol{r}_{\text {target }}-\boldsymbol{r}_{\text {monitor }}\right) \cdot \boldsymbol{n}}{\boldsymbol{V}_{s w} \cdot \boldsymbol{n}}$.

Here, $\boldsymbol{r}_{\text {target }}$ is the position of the target (typically the upstream magnetopause position at around $X_{\mathrm{GSE}}=10-15 R_{E}$ ), $\boldsymbol{r}_{\text {monitor }}$ is the position of the solar wind monitor, in our case the ACE spacecraft orbiting the L1 libration point, and $\boldsymbol{V}_{s w}$ is the measured solar wind velocity.

There are several methods to infer the boundary normal of a plasma boundary. The typical IMF orientation is aligned along the Parker spiral, approximately $45^{\circ}$ to the solar wind flow direction. Horbury et al. (2001a) calculated propagation times using both normals parallel and perpendicular to the Parker spiral, but found that these assumptions often gave poorer results than simply assuming a flat delay. More sophisticated methods require in-situ measurements and then trying to estimate the normal from multi spacecraft timing or gradient methods (e.g. Horbury et al., 2001b; Knetter et al., 2004), or use the local field or plasma measurements from a single spacecraft (see e.g. overviews in Sonnerup et al., 2006; Volwerk, 2006). 


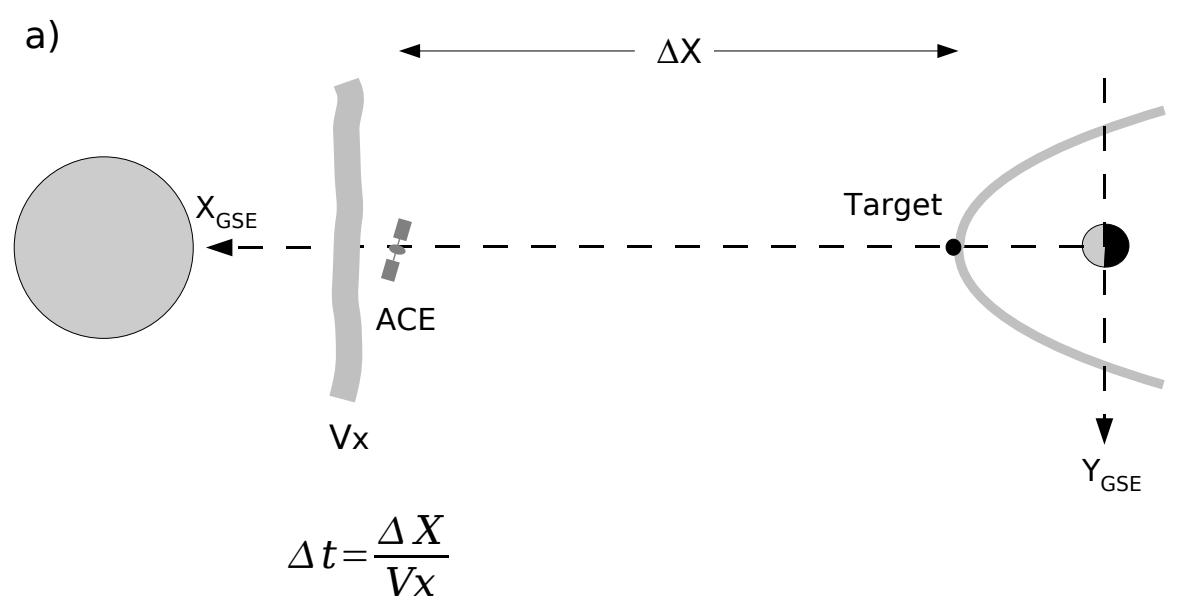

b)

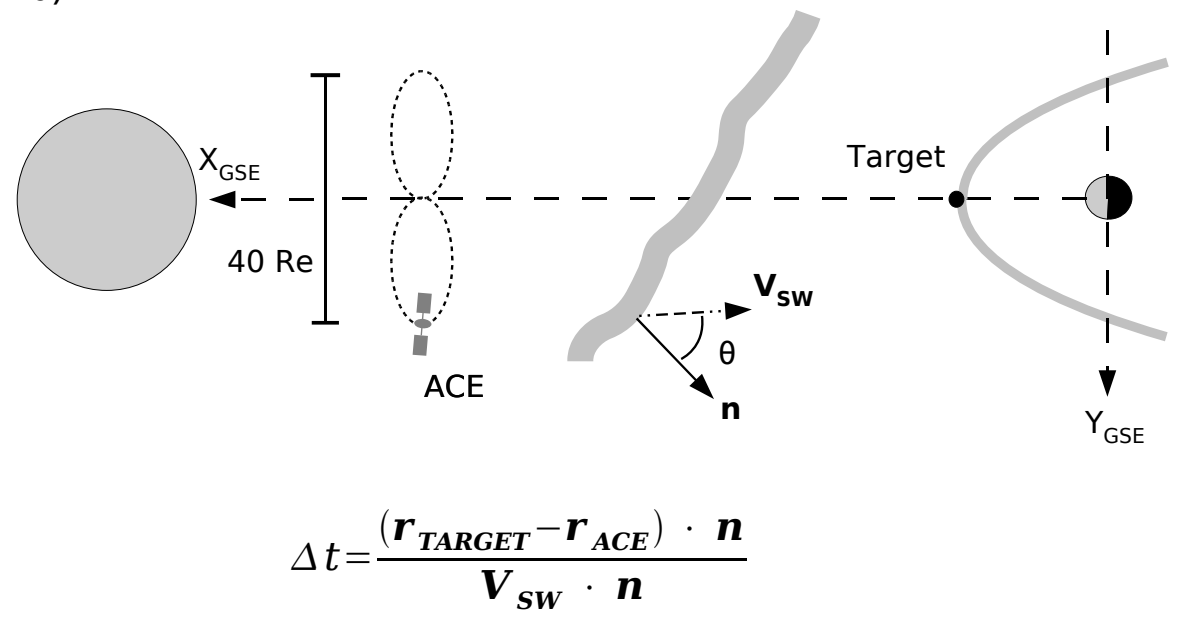

Fig. 1. Illustration of the two principal methods to calculate the solar wind propagation delay. (a) Flat delay: A planar structure is assumed to propagate with a constant velocity along the Sun-Earth line. Both the solar wind monitor (ACE) and the target (typically the Earth's upstream magnetopause) are assumed to lie on the Sun-Earth line. (b) The real position of the solar wind monitor as well as the orientation of the IMF phase front, represented by its boundary normal $\boldsymbol{n}$, and the actual solar wind velocity vector, $\boldsymbol{v}_{s} w$ are taken into account. $\theta$ is the angle between the phase front normal and the solar wind velocity (in Weimer et al., 2003, the angle $\theta$ is measured between the normal and the Sun-Earth line).

\subsubsection{Discontinuity orientation from cross product}

The cross product method can be used to get the orientation of a tangential discontinuity (TD - see e.g. Colburn and Sonett, 1966; Smith, 1973), i.e. a discontinuity where there is no net plasma flow across the discontinuity $(\langle\boldsymbol{V}\rangle \cdot \boldsymbol{n}=0)$, and where the average magnetic field is tangential to the discontinuity $(\langle\boldsymbol{B}\rangle \cdot \boldsymbol{n}=0)$. If these conditions are satisfied, or nearly satisfied, an estimate of the boundary normal is given by:

$\boldsymbol{n}_{\mathrm{cross}}=\frac{\left\langle\boldsymbol{B}_{1}\right\rangle \times\left\langle\boldsymbol{B}_{2}\right\rangle}{\left|\left\langle\boldsymbol{B}_{1}\right\rangle \times\left\langle\boldsymbol{B}_{2}\right\rangle\right|}$

where $\left\langle\boldsymbol{B}_{1}\right\rangle$ and $\left\langle\boldsymbol{B}_{2}\right\rangle$ are the average magnetic field upstream respectively downstream of the discontinuity.
In our study, we have calculated the downstream average $\left\langle\boldsymbol{B}_{1}\right\rangle$ from 10 magnetic field samples ending approximately $3.5 \mathrm{~min}$ before the center of the discontinuity. Likewise, the upstream average $\left\langle\boldsymbol{B}_{2}\right\rangle$ is calculated from 10 samples starting approximately $3.5 \mathrm{~min}$ after. These time intervals are indicated in the example shown in Fig. 2.

\subsubsection{Minimum variance of the magnetic field - MVAB}

Minimum variance of the magnetic field (MVAB - see e.g. Sonnerup and Cahill, 1967; Sonnerup and Scheible, 1998, and references therein) is perhaps the most frequently used method to obtain the orientation of a discontinuity. MVAB is based on a one-dimensional model of a current sheet. From 
the magnetic field vector measurements during the transversal of a discontinuity, one tries to find the apriori unknown direction in which the magnetic field has no variance. In practice, however, this ideal case does not exist, and one seeks to find the direction with minimum variance of the magnetic field. Mathematically, this is achieved by first constructing a magnetic covariance matrix, $\mathbf{M}_{\nu \mu}$, from the measurements, and thereafter finding the eigenvectors and eigenvalues of this matrix. In the present work, we have used a covariance matrix of the form

$\mathbf{M}_{\nu \mu}=\left\langle B_{\mu} B_{v}\right\rangle-\left\langle B_{\mu}\right\rangle\left\langle B_{v}\right\rangle$

where $\langle\ldots\rangle$ denotes averaging over a number of measurements. This corresponds to the standard covariance matrix introduced in Sonnerup and Cahill (1967). Other types of covariance matrices are also conceivable. For example, Siscoe et al. (1968) used a simplified covariance matrix of the form

$\mathbf{M}_{\nu \mu}=\left\langle B_{\mu} B_{v}\right\rangle$

for the study of discontinuities in the IMF observed by the Mariner 4 spacecraft. A similar approach was used by Snekvik et al. (2007) to establish the orientation of the tail current sheet. Weimer et al. (2003) initially used a covariance matrix of the form

$\mathbf{M}_{\nu \mu}=\left\langle B_{\mu} B_{\nu}\right\rangle-N\left\langle B_{\mu}\right\rangle\left\langle B_{\nu}\right\rangle$

where $N$ is the number of samples used to form the averages. As pointed out in a later correction (Weimer, 2004), this matrix is dominated by the mean magnetic field, and results in negative eigenvalues. The eigenvector corresponding to the smallest, non-negative eigenvalue is nearly orthogonal (but not completely - see discussions in Bargatze et al., 2005, and Haaland et al., 2006) to the mean magnetic field, and thus similar to the cross product method described above. The variance matrix used by Siscoe et al. (1968) and Snekvik et al. (2007) has a similar property.

Weimer et al. (2003) provided a recipe-like procedure for establishing the phase front orientation; first, a short time interval of approximately $8 \mathrm{~min}$ was used to construct a covariance matrix. Thereafter, an eigenanalysis was performed on this matrix. If the resulting eigenvalue ratio was poor (i.e. $\lambda_{\text {int }} / \lambda_{\min } \leq 10$ ), the calculation was discarded and a longer time interval of approximately $28 \mathrm{~min}$ was tried. For the long time interval, Weimer et al. (2003) also reduced the eigenvalue criteria, so that a ratio $\lambda_{\text {int }} / \lambda_{\min } \geq 2$ was accepted. If both time intervals failed, the previous valid normal was used for the delay calculation.

However, since we use a different covariance matrix, we cannot directly adapt the criteria from Weimer et al. (2003). In our calculations we have therefore used a fix 7-min interval centered around the magnetic field rotation to establish the phase front normal. This time interval is marked " $A$ " in Fig. 2. The choice of $7 \mathrm{~min}$ seems arbitrary, but was found to
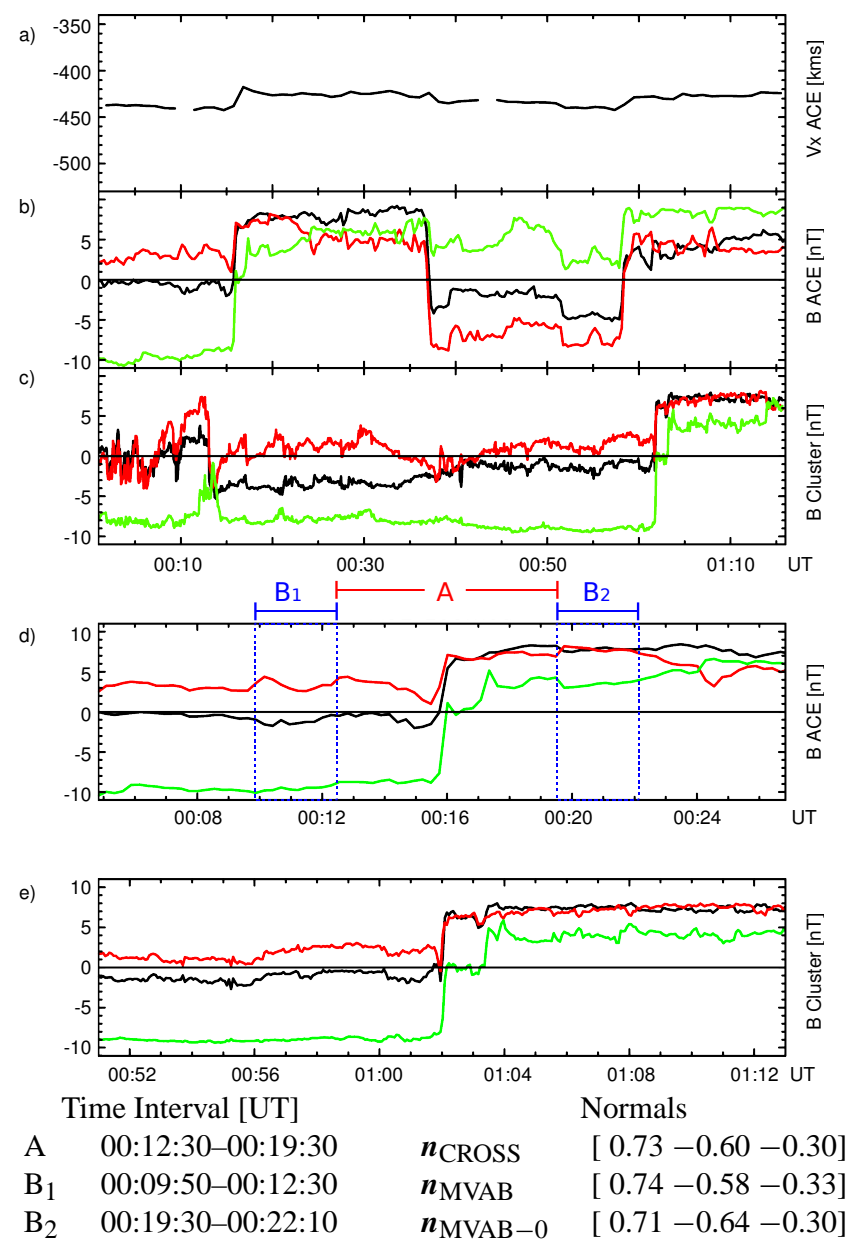

Fig. 2. Example of an IMF discontinuity observed by both ACE and Cluster on 30 March 2003. The panels show: (a) the X component of the solar wind speed measured by ACE. (b) magnetic field at ACE, (c) magnetic field at Cluster. (d, e) Same as panels (b) and (c), but zoomed in and centered around the main magnetic field rotation. In panel (d), the marked interval $A$ is the time segment used to calculate the IMF orientation as described in the Sects. 3.2.2 and 3.2.3. Similarly, $B_{1}, B_{2}$ are the corresponding upstream and downstream time intervals used for the cross product calculation - see Sect. 3.2.1. Black, red and green line colors indicate the X, Y, respectively $Z_{\mathrm{GSE}}$ components of the magnetic fields. The bottom part of the figure shows the exact time intervals used and the normals obtained for this particular case.

be a good compromise which ensures sufficient data points within and on both sides of the discontinuity. With 16-s time resolution in the ACE magnetic field observations, this interval contains 28-30 samples.

\subsubsection{Constrained minimum variance - MVAB-0}

If one has apriori knowledge about the nature of a discontinuity, it may be desirable to impose constraints to the minimum variance analysis. For example, an ideal TD has zero 
magnetic field along the normal. One can then constrain perform the analysis so that the predicted normal, $\boldsymbol{n}$, is guaranteed to be perpendicular to the direction of the average magnetic field $\boldsymbol{b}=<B>/|<B>|$. Such a constraint can easily be imposed to the variance analysis by replacing the above covariance matrix, $\mathbf{M}_{v \mu}$, by the projection $\mathbf{Q}^{\prime}=P_{i k} M_{v \mu} P_{n j}$, where the projection matrix is given by

$P_{i j}=\delta_{i j}-b_{i} b_{j}$

where $\delta_{i j}$ is the delta operator ( $\delta_{i j}=1$ for $i=j, 0$ otherwise). The eigenvectors of $\mathbf{Q}^{\prime}$ now have a different meaning; since we introduce a known quantity (the vector $\boldsymbol{b}$ ), the lowest eigenvalue will be zero, whereas its eigenvector, $\boldsymbol{X}_{3}=\boldsymbol{b}$. The eigenvector $\boldsymbol{X}_{2}$, corresponding to the lowest, non-zero eigenvalue will now be the normal predictor, and the third eigenvector completes the right handed, orthogonal system.

An alternative procedure to obtain a discontinuity normal orthogonal to the mean magnetic field is given by Bargatze et al. (2005). Their method uses a variance matrix based on $\boldsymbol{B}_{\perp}(t)=\boldsymbol{B}(t)-\boldsymbol{B}_{\|}$, where $\boldsymbol{B}_{\|}$is the magnetic field parallel to the average magnetic field of the 8 or $28 \mathrm{~min}$ time interval mentioned in Weimer et al. (2003).

Experience has shown that constrained variance analysis, often referred to as MVAB-0, provides more stable results, also for discontinuities of Alfvénic nature (Sonnerup et al., 2006).

In a survey, Knetter et al. $(2003,2004)$ applied multispacecraft timing methods to a number of discontinuities observed by Cluster in the solar wind, and concluded that most of the observed discontinuities could be classified as TDs. Also, in a recent publication, Weimer and King (2008), adapted the MVAB-0 method, and performed a thorough check of the method, and basically confirmed its usefulness for propagation delay calculations. Although the abundances of rotational and tangential discontinuities in the solar wind have been debated (Neugebauer et al., 1984; Tsurutani and Ho, 1999; Ridley, 2000; Knetter et al., 2004; Neugebauer, 2006), it seems that the assumption that solar wind discontinuities are TDs is justified for this purpose.

\subsection{Error sources and quality criteria}

The "observed" delay in our study is established through visual inspection of the data, and trying to line up the ACE and Cluster magnetic field measurements as shown in Fig. 2. Since most of our events are manifested as distinct and sharp rotations in the magnetic field, we estimate the determination of the observed propagation time to be accurate to within \pm 1 min or less. With only one solar wind monitor available, we cannot say anything about the planarity of the discontinuities, so in the following, we assume that the observed discontinuities are planar.

For the model calculations, the most critical parameter is the orientation of the discontinuity. For MVAB based methods, analytical expressions for the errors in the form of er- ror ellipses around the normal exist (e.g. Lepping and Behannon, 1980; Khrabrov and Sonnerup, 1998; Sonnerup and Scheible, 1998). A similar approach can in theory also be implemented for the cross product method. However, error estimates of this character are purely statistical and mainly depends on the number of samples used to establish the normal. Errors due to e.g. breakdown of the underlying model assumptions are not taken into account by such error estimates. We have therefore not performed any detailed error analysis of this sort in our study. However, we are able to formulate a set of quality criteria which can be used to determine whether a particular method makes sense for a specific event.

For the MVAB based results, the ratio between the intermediate and minimum eigenvalue provides a rough quality control of the result. As a rule of thumb, an eigenvalue ratio, $\lambda_{\text {int }} / \lambda_{\min } \geq 10$ has often been used as a criteria for a valid normal determination, although this is rarely achieved without fine tuning of the time interval used for analysis. In our data set, and using the variance matrix as described in Sect. 3.2.2, less than $20 \%$ of of the discontinuities had an eigenvalue ratio $\lambda_{\text {int }} / \lambda_{\min } \geq 10$. We have therefore required an minimum eigenvalue ratio of $\lambda_{\text {int }} / \lambda_{\min } \geq 3$.

It should be emphasized, however, that a high eigenvalue ratio in itself is no guarantee for a correct normal estimation. Normals obtained from minimum variance and normals obtained from multi spacecraft methods can in some cases be widely different, despite high eigenvalue ratios (Knetter et al., 2004; Haaland et al., 2004; Sonnerup et al., 2008). Also, the eigenvalue ratio only describes the statistical uncertainty in the eigenvector determination. As with some of the error estimates, the eigenvalue ratio does not account for breakdown of the model assumptions.

For the constrained minimum variance, the lowest eigenvalue is per definition zero, and the only sensible eigenvalue ratio is $\lambda_{\max } / \lambda_{\text {int }}$. Since the maximum variance direction is typically well defined for a 1-D or 2-D structure, this ratio is usually much higher (typically a factor 10 or more) than the above $\lambda_{\text {int }} / \lambda_{\min }$ ratio. In our data set, the majority of the events had eigenvalue ratios $\lambda_{\max } / \lambda_{\text {int }} \geq 60$, so we decided to require a minimum eigenvalue ratio of 10 for this quality parameter.

For the cross product methods, we checked the angular difference, $\phi$, between the upstream and downstream magnetic field (i.e. the field rotation). Values around $0^{\circ}$ or $180^{\circ}$ indicate parallel or antiparallel fields, and thus a poorly determined cross product. In our study, we have required that the orientation of $\left\langle\boldsymbol{B}_{1}\right\rangle$ and $\left\langle\boldsymbol{B}_{2}\right\rangle$ differs at least $30^{\circ}$. This criteria is similar to the definition used by Lepping and Behannon (1986).

In addition to the above, we also required that the calculated normal should be within $\theta= \pm 70^{\circ}$ of the solar wind velocity direction (see Fig. 1). A similar criteria was used by Weimer et al. (2003). 


\section{Results}

During the period February 2001 to April 2007, we found a total of 198 clear discontinuities that could be unambiguously identified at both ACE and Cluster. For each event, we first plotted the ACE magnetic field, solar wind velocity, the corresponding Cluster magnetic field, and recorded the position of ACE relative to Cluster. Thereafter, we calculated the time delays as well as the quality parameters for the four methods.

\section{$4.1 \quad$ Example}

Figure 2 shows an example of a single event. On 30 March 2003, the ACE spacecraft observed a series of distinct magnetic field rotations. At about 00:16 UT the IMF direction turns from a predominantly southward direction to a more Parker spiral like orientation. Prior to the rotation, the IMF is fairly stable with a solar wind velocity of about $435 \mathrm{~km} \mathrm{~s}^{-1}$. The same magnetic field rotation is seen at Cluster about 01:02 UT, i.e. around $46 \mathrm{~min}$ later. Cluster was located around $14 R_{E}$ upstream, and the average separation in Y-direction between Cluster and ACE was only about $13 R_{E}$ in this case.

The calculated normals from the three methods MVAB, MVAB- 0 and cross product are very similar, and have a significant $\mathrm{X}_{\mathrm{GSE}}$ component. All methods, including the flat delay method, were able to predict the propagation time from ACE to Cluster to within $4 \mathrm{~min}$ for this event. The poorest performance was the flat delay, which predicted a propagation time of $51 \mathrm{~min}$ in this case, whereas the best prediction was obtained with the MVAB- 0 method, which predicted the arrival time to within one minute.

Although our selection of events may be biased, this example is by no means atypical; due to the high velocity of the solar wind compared to the available time resolution of the data, most events are manifested as sharp transitions in the IMF which allows for a fairly precise determination of the real time delay.

\subsection{Statistical deviations between observed and predicted arrival times}

In the following, we discuss the deviations between predicted and observed arrival times of the discontinuities in a statistical sense. For all 198 events, we calculate a timing error for each method, defined as $\Delta t=t_{\text {model }}-t_{\text {observed }}$, and try to find correlations with quality parameters, IMF orientation and spacecraft separation distances.

Figure 3 shows the relative distribution of the timing errors for the four models. To show the effect of the quality parameters discussed above, we also show the distributions from a filtered data set (lower panels). Depending on method, a number of events fall below our quality criteria.
We did not apply any filters to the flat delay, so this is equal in the top and bottom panels. As seen from the distribution, the predicted arrival time of the the discontinuities at Cluster are within $10 \mathrm{~min}$ in most cases, and a substantial number of events even arrive at Cluster within $\pm 5 \mathrm{~min}$ of the time predicted by the flat delay method.

For the cross product method, the majority of discontinuities arrive at Cluster within $\pm 5 \mathrm{~min}$ of the predicted time, regardless of whether we consider the filtered or unfiltered set. For the filtered data set, an arrival accuracy of $\pm 2 \mathrm{~min}$ is obtained for almost $30 \%$ of the cases.

In our data set, the MVAB method perform worse than the simple delay of cross product method. Still, most of the discontinuities arrive within $\pm 10 \mathrm{~min}$ of the predicted time. Removing events which do not satisfy the quality criteria, results improves the relative accuracy, and more than $50 \%$ of the cases arrives within \pm 5 min.

For the constrained minimum variance method, $65 \%$ of the cases have a timing accuracy of $\pm 5 \mathrm{~min}$ or better, and more than $30 \%$ have an arrival accuracy of \pm 2 min or less. For the filtered dataset, the results are even better - more than $35 \%$ of the events arrive within the $\pm 2 \mathrm{~min}$ of the predicted time, and $82 \%$ arrive within \pm 5 min of the predicted time.

In summary, the constrained minimum variance analysis gives the best performance, but the cross product also provides a fairly accurate estimate of the arrival time in most cases.

\subsubsection{Spacecraft separation effect}

One of the motivations for Weimer et al. (2003) was to devise a method that takes into account the lateral displacement, i.e. primarily $\mathrm{Y}_{\mathrm{GSE}}$ separation between the monitor and the target. In Fig. 4 we have plotted the timing errors of each method as function of the spacecraft separation distance in y-direction: $\Delta Y=\mathrm{ACE} \mathrm{Y}_{\mathrm{GSE}}-$ Cluster $\mathrm{Y}_{\mathrm{GSE}}$. The ACE orbit alone takes the spacecraft out to approximately $40 R_{E}$ away from the Sun-Earth line, whereas for the seasons we have studied, Cluster is maximum $8 R_{E}$ away from the Sun-Earth line. Both Weimer et al. (2003) and Horbury et al. (2001a) additionally used the Wind spacecraft, and partly the Interplanetary Monitoring Platform (IMP-8) and the Geotail spacecraft, so their data set and discussion had significantly larger lateral separations.

As shown in Fig. 4, the best prediction of the arrival time, i.e. a $\Delta t \simeq 0$ is typically obtained when both ACE and Cluster are located roughly on the same $\mathrm{Y}_{\mathrm{GSE}}$ position. In particular, the flat delay method shows large spreads in the results when the lateral separation is more than $40 R_{E}$. For large positive separations $\Delta t$ tends to be negative, (i.e. the calculated time delay is smaller than the observed), whereas the opposite is the case for large negative distances. This is as expected for a typical Parker spiral like orientation of the IMF.

From Fig. 4, it also appears that some of the events with large timing errors are common to several methods (e.g. the 

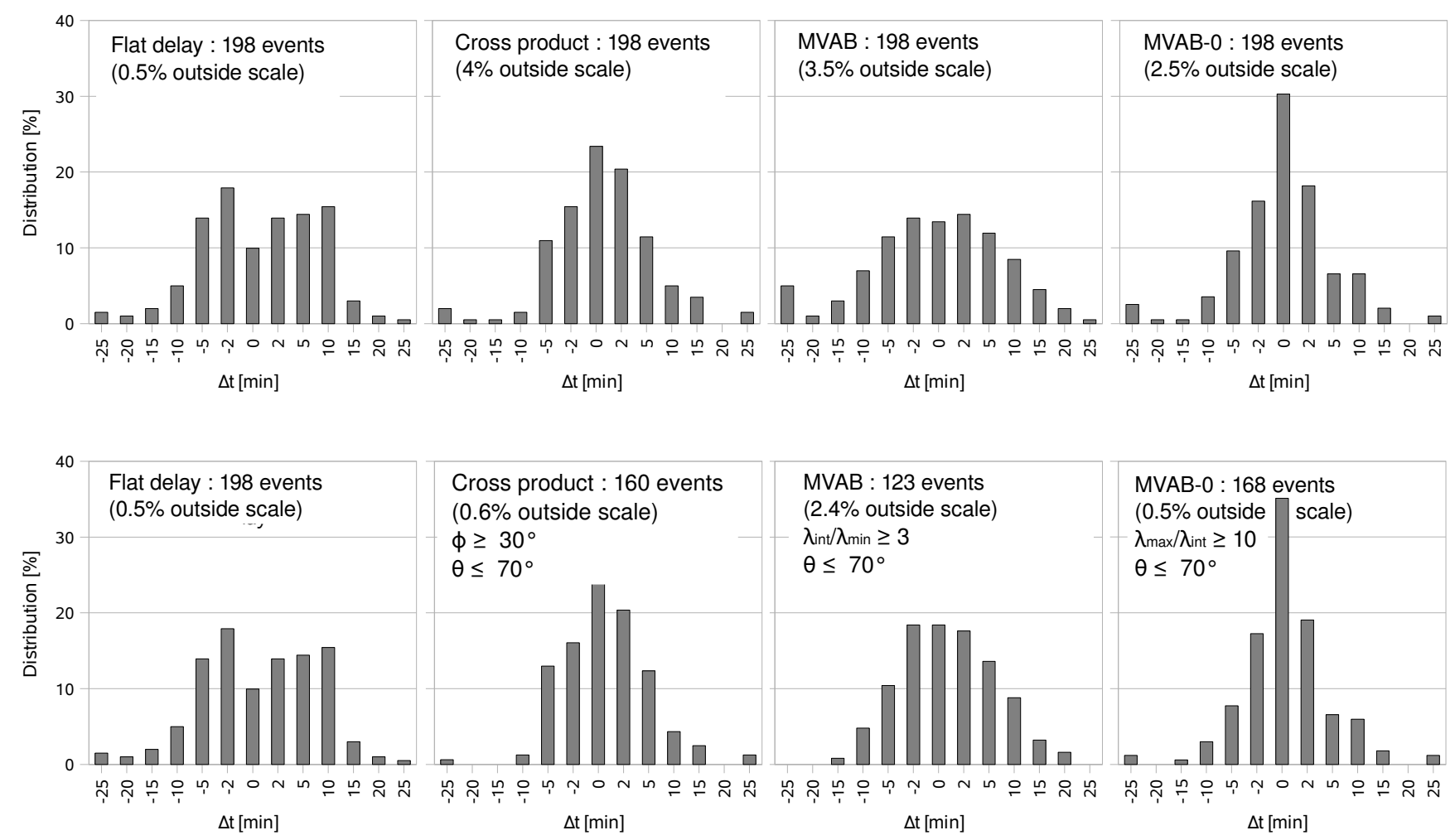

Fig. 3. Distributions of timing errors for each of the four tested methods. Top panels: results from the full data set, containing 198 events. Bottom panels: results from the filtered data set, i.e. events not satisfying the quality criteria described in Sect. 3.3 have been removed. The horizontal axes indicate the time differences $\Delta t=t_{\text {model }}-t_{\text {observed }}$ for each method, and the vertical axes, common to all panels in that row, show the relative distribution within that $\Delta t$ range. Note that the horizontal axes are non-linear; the center bin (marked 0$)$ means $\pm 0-2$ min, the next bin $\pm 2-5 \mathrm{~min}$, thereafter $\pm 5-10 \mathrm{~min}$ etc. Also, the horizontal scale is limited to $\pm 25 \mathrm{~min}$, but a few events from each method had larger discrepancies. These are indicated in each panel as (\% outside scale).

two data points with $\Delta t \simeq 22 \mathrm{~min}$ seen in the panels for cross product and MVAB-0 results). This could either mean that both methods fail to give correct orientations, or that other effects such as non-planarity or scale sizes of the discontinuity also affect the results.

In this sense, the results of Tsurutani et al. (2005) are somewhat exceptional. Although their $\Delta Y$ were in the range 28-39 $R_{E}$, the observed arrival times were less than a minute from the flat delay predictions.

\subsubsection{Influence of IMF orientation}

A critical factor for time shift estimations relying on the MVAB, MVAB-0 and cross product method is the discontinuity orientation. The top panel of Fig. 5 shows the timing error as function of the $\theta$ angle, which is the angle between the discontinuity normal and the solar wind velocity (also illustrated in the lower panel of Fig. 1). A large angle indicate a slant discontinuity orientation, whereas the typical Parker spiral like orientation would give a $\theta$ angle around $45^{\circ}$. For all three methods, a deterioration is seen for large angles.
To check the reliability of our normal estimations, we checked the correlation between the quality criteria described in Sect. 3.3 and the timing errors:

For the minimum variance methods, we checked the dependence on the eigenvalue ratios $\lambda_{\text {int }} / \lambda_{\min }$ for MVAB and $\lambda_{\max } / \lambda_{\text {int }}$ for MVAB-0. The bottom panel of Fig. 5 shows the timing errors as function of these two ratios. As explained in Sect. 3.3, the eigenvalue ratios for MVAB and MVAB- 0 are not directly comparable; the MVAB-0 eigenvalue ratio $\lambda_{\max } / \lambda_{\text {int }}$ is typically a factor 10 higher than the ratio $\lambda_{\text {int }} / \lambda_{\min }$ from MVAB. We have therefore used two horizontal axes and color coded the results in the lower panel of Fig. 5. The plot shows a clear dependence; low eigenvalue ratios, which typically indicate poorly determined normals, gives a larger difference between the predicted and observed time delays. From the plot, one would conclude that an eigenvalue ratio $\lambda_{\text {int }} / \lambda_{\min } \geq 10$ for MVAB and $\lambda_{\max } / \lambda_{\text {int }} \geq 100$ would be desireable for optimal results. Unfortunately, very few of our events have such large eigenvalue ratios.

For the cross product method, we also checked the correlation between the timing error and the $\phi$ angle (i.e. the field rotation between the upstream and downstream B-field). 

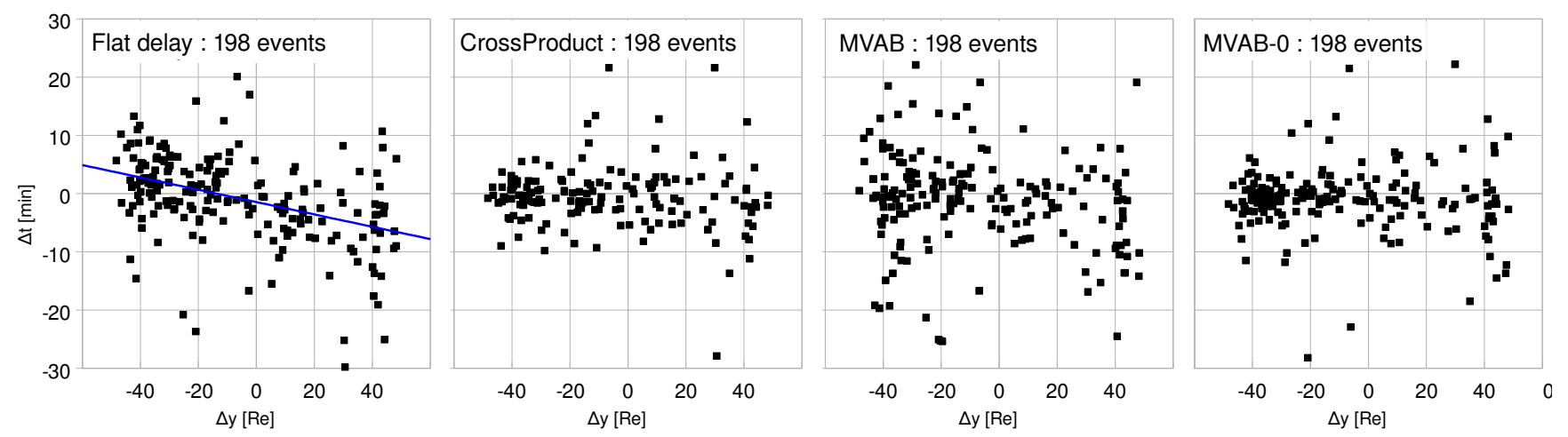

Fig. 4. Arrival time errors as function of the separation between ACE and Cluster in the $\mathrm{Y}_{\mathrm{GSE}}$ direction. The vertical axis, common to all panels, shows the time differences $\Delta t=t_{\text {model }}-t_{\text {observed }}$, and the horizontal axes show the $\mathrm{Y}_{\mathrm{GSE}}$ separation between the ACE and Cluster spacecraft, $\Delta Y=Y_{\mathrm{ACE}}-Y_{\mathrm{CLUSTER}}$ for each method. Since the Cluster orbit in our data set has only has a maximum $\mathrm{Y}_{\mathrm{GSE}}$ position of $\pm 8 R_{E}$, this plot can roughly be interpreted as the dependence of the ACE $\mathrm{Y}_{\mathrm{GSE}}$ position. The blue line in the panel for flat delay indicates the linear trend calculated from the data points.

However, we could not find any correlation here, and only a few events had field rotations of less then $30^{\circ}$.

\subsection{Cases with no discontinuities}

So far, we have focused on time intervals which contain a distinct discontinuity, observed as a clear rotation in the magnetic field. Despite the frequent occurrence of discontinuities in the solar wind, a more typical situation is a fairly stable IMF, often aligned along the Parker spiral. As pointed out above, the knowledge of the IMF conditions at the Earth's magnetopause is also important for such cases, and is one of the key aspects addressed by the methods of (Weimer et al., 2003; Weimer and King, 2008).

To test the ability of the various methods to predict propagation times under such conditions, we repeated the calculations from Sect. 3, but now using a time interval 7 min later. This interval typically does not contain any distinct discontinuity (although it may). In most of our cases, the change in the solar wind velocity between the upstream and downstream of the discontinuity was small, and the changes in separation between ACE and Cluster within 7 min are also negligible. The flat delay results are therefore similar, and the true propagation, $t_{\mathrm{obs}}$ from the data set with distinct discontinuities can therefore still be used as a reference for benchmarking. An alternative method would have been to pick out random time intervals for this test, and tried to establish the true time delay from e.g. cross correlation, but we did not try this out.

In this displaced time interval, one would expect that the methods relying on the orientation would fail or deteriorate since there is no clear rotation of the field any more, and the assumptions implied by both minimum variance (a quasi 1D current sheet) and the cross product method (a tangential discontinuity) would fail.
This is also exactly what happens to our data set. Our quality criteria for the cross product $\left(\phi \geq 30^{\circ}-\right.$ see Sect. 3.3) fails for more than half of the events, and a reliable cross product normal can only be obtained for 63 of our 198 events. Of these, arrival times within \pm 5 min are obtained for 41 events, compared to 84 events for a simple flat delay. The MVAB based propagation times also performs worse for this data set. The eigenvalue ratios are often very low which indicate poorly determined normals.

The performance of the MVAB- 0 method is also reduced, but this methods still gives the the overall best estimate of the propagation delay. Approximately $71 \%$ of the calculated propagation times are within $10 \mathrm{~min}$ of the observed time, but a few of the predicted times are very much longer than the observed. However, the flat delay method, not relying on the IMF orientation performs equally well as for the data set with discontinuities. With almost $70 \%$ of the cases arriving within $10 \mathrm{~min}$ of the observed times, it performs only marginally worse than the MVAB- 0 results.

\section{Summary and conclusions}

We have done a statistical study of the propagation times of IMF discontinuities between the ACE solar wind monitor orbiting the L1 libration point and the Cluster quartet of spacecraft close to the Earth's bow shock. The two spacecraft are separated between 0 and $\pm 48 R_{E}$ in the $\mathrm{Y}_{\mathrm{GSE}}$ direction.

For 198 distinct discontinuities, we calculated the time shift as predicted by four different models, and compared this to the observed time delay.

The results can be summarized as follows:

- The most precise determination of the arrival of a discontinuity at a target near the Earth's magnetopause is 

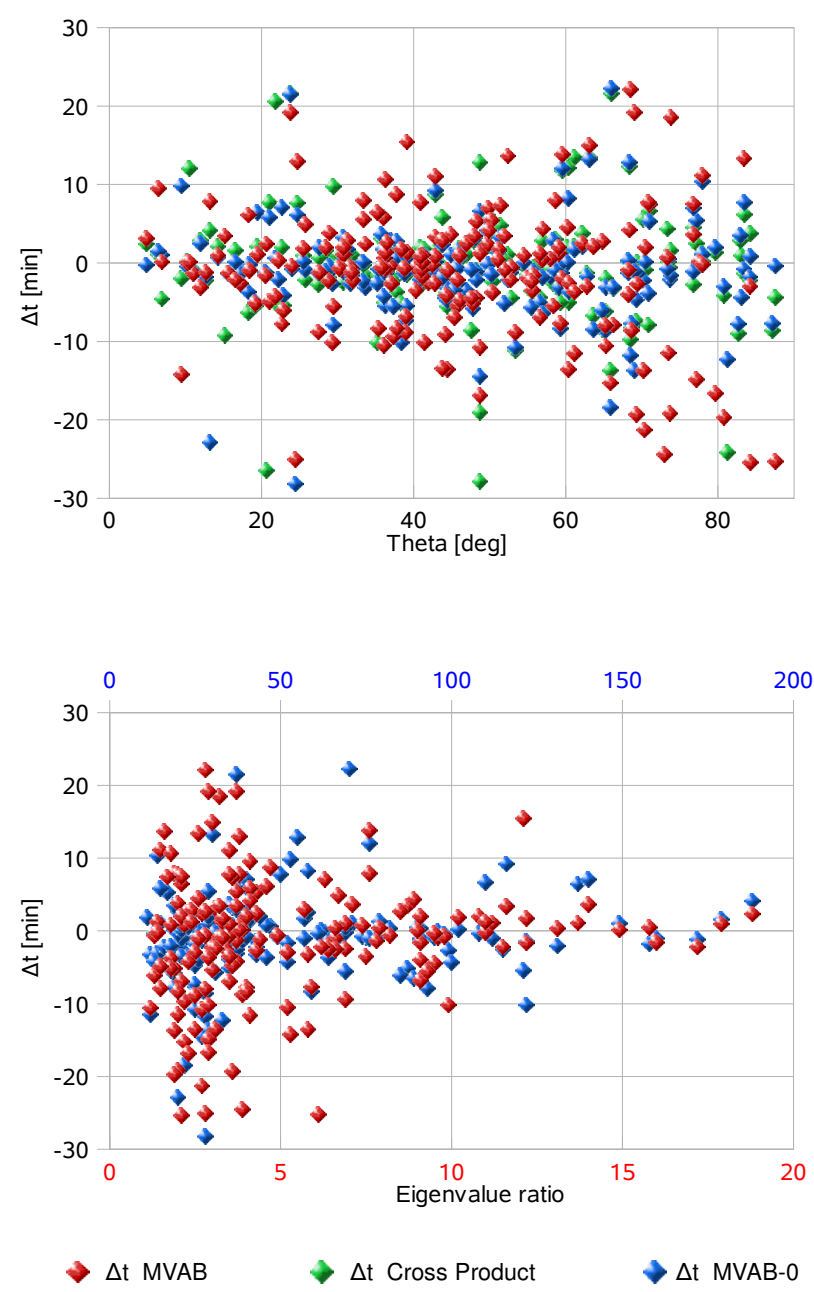

Fig. 5. Top panel : Difference between observed and predicted arrival times as function of the angle $\theta$ (the angle between the discontinuity normal and the solar wind velocity - see also Fig. 1). Bottom panel: Difference between observed and predicted arrival times as function of eigenvalue ratio for the minimum variance based methods. The vertical axes in both panels show the time differences $\Delta t=t_{\text {model }}-t_{\text {observed }}$. In the lower panel, red data points, and the lower vertical axis indicate the $\lambda_{\text {int }} / \lambda_{\min }$ eigenvalue ratio for MVAB, whereas the blue symbols and the upper horizontal axis indicate the corresponding ratio $\lambda_{\max } / \lambda_{\text {int }}$ for the MVAB- 0 method.

achieved if the orientation of the discontinuity is taken into account.

- For the data set discussed here, the best predictions of arrival times of discontinuities at the Earth's magnetopause are obtained if the orientation of the discontinuity is obtained from constrained minimum variance analysis of the magnetic field as suggested by Weimer et al. (2003).
- The arrival predictions using flat delay deteriorates, but not significantly, if the solar wind monitor is far away from the Sun-Earth line. This argument may not be very important in cases where the ACE spacecraft with its maximum $\pm 40 R_{E}$ displacement from the Sun-Earth line, but should be kept in mind if the IMF observations are taken other solar wind monitors with larger lateral displacements are used.

- In our data set, the MVAB-0 based method works fairly well even for cases without a distinct discontinuity, and provide more reliable predictions than the other methods.

- For cases without any clear discontinuities, the prediction of the arrival times becomes less precise for both the cross product method and the minimum variance method.

- Despite the fact that propagation delay calculations based on the constrained minimum variance analysis performed better then the other methods, there are some drawbacks with the method. Compared to a simple flat delay calculation, which may be "good enough", it is computational much more complex.

Acknowledgements. This project was initiated as a student project at the COSPAR Regional Workshop for Space Physicists from Central and Eastern Europe, held in Sinaia, Romania, 4-16 June 2007. B. Mailyan thanks the Centre national de la recherche scientifique (CNRS), France for financial support to attend and present these results at the STAMMS-2 conference, held in Orleans, France in September 2007. Parts of the data analysis were done with the QSAS science analysis system provided by the United Kingdom Cluster Science Centre (Imperial College London and Queen Mary, University of London) supported by the United Kingdom Science and Technology Facilities Council (STFC). We also thank the International Space Science Institute, Bern, Switzerland for providing computer resources and infrastructure for data exchange. ACE data were obtained from the Coordinated Data Analysis Web (CDAWeb - see http://cdaweb.gsfc.nasa.gov/about.html).

Topical Editor R. Nakamura thanks D. Weimer and another anonymous referee for their help in evaluating this paper.

\section{References}

Balogh, A., Carr, C. M., Acuña, M. H., Dunlop, M. W., Beek, T. J., Brown, P., Fornacon, K. H., Georgescu, E., Glassmeier, K. H., Harris, J., Musmann, G., Oddy, T., and Schwingenschuh, K.: The Cluster Magnetic Field Investigation: Overview of in-flight performance and initial results, Ann. Geophys., 19, 1207-1217, 2001 , http://www.ann-geophys.net/19/1207/2001/.

Bargatze, L. F., McPherron, R. L., Minamora, J., and Weimer, D.: A new interpretation of Weimer et al.'s solar wind propagation delay technique, J. Geophys. Res., 110, 7105, doi: 10.1029/2004JA010902, 2005. 
Colburn, D. S. and Sonett, C. P.: Discontinuities in the Solar Wind, Space Sci. Rev., 5, 439-506, doi:10.1007/BF00240575, 1966.

Dungey, J. R.: Interplanetary magnetic field and the auroral zones, Phys. Rev. Lett, 6, 47-48, 1961.

Förster, M., Paschmann, G., Haaland, S. E., Quinn, J. M., Torbert, R. B., Vaith, H., and Kletzing, C. A.: High-latitude plasma convection from Cluster EDI: variances and solar wind correlations, Ann. Geophys., 25, 1691-1707, 2007, http://www.ann-geophys.net/25/1691/2007/.

Gombosi, T. I., Dezeeuw, D. L., Groth, C. P. T., and Powell, K. G.: Magnetospheric Configuration for Parker-Spiral IMF Conditions: Results of A 3D AMR MHD Simulation, Adv. Space Res., 26, 139-149, 2000.

Haaland, S., Sonnerup, B. U. Ö., Dunlop, M. W., Balogh, A., Hasegawa, H., Klecker, B., Paschmann, G., Lavraud, B., Dandouras, I., and Rème, H.: Four-Spacecraft Determination of Magnetopause Orientation, Motion and Thickness: Comparison with Results from Single-Spacecraft Methods, Ann. Geophys., 22, 1347-1365, 2004,

http://www.ann-geophys.net/22/1347/2004/.

Haaland, S., Paschmann, G., and Sonnerup, B. U. Ö.: Comment on "A new interpretation of Weimer et al.'s solar wind propagation delay technique" by Bargatze et al., J. Geophys. Res., 111, 61026106, doi:10.1029/2005JA011376, 2006.

Haaland, S. E., Paschmann, G., Förster, M., Quinn, J. M., Torbert, R. B., McIlwain, C. E., Vaith, H., Puhl-Quinn, P. A., and Kletzing, C. A.: High-latitude plasma convection from Cluster EDI measurements: method and IMF-dependence, Ann. Geophys., 25, 239-253, 2007,

http://www.ann-geophys.net/25/239/2007/.

Horbury, T. S., Burgess, D., Fränz, M., and Owen, C. J.: Prediction of Earth arrival times of interplanetary southward magnetic field turnings, J. Geophys. Res., 106, 30 001-30 009, 2001a.

Horbury, T. S., Burgess, D., Fränz, M., and Owen, C. J.: Three spacecraft observations of solar wind discontinuities, Geophys. Res. Lett., 28, 677-680, doi:10.1029/2000GL000121, $2001 \mathrm{~b}$.

Hudson, P. D.: Discontinuities in an anisotropic plasma and their identification in the solar wind, Planet. Space Sci., 18, 16111622, doi:10.1016/0032-0633(70)90036-X, 1970.

Khrabrov, A. V. and Sonnerup, B. U. Ö.: Error estimates for minimum variance analysis, J. Geophys. Res., 103, 6641, 1998.

Knetter, T.: A new perspective of the solar wind micro-structure due to multi-point observations of discontinuities, Ph.D. thesis, University of Cologne, Germany, 2004.

Knetter, T., Neubauer, F. M., Horbury, T., and Balogh, A.: Discontinuity observations with Cluster, Adv. Space. Res., 32, 543-548, doi:10.1016/S0273-1177(03)00335-1, 2003.

Knetter, T., Neubauer, F. M., Horbury, T., and Balogh, A.: Fourpoint discontinuity observations using Cluster magnetic field data: A statistical survey, J. Geophys. Res., 109, 6102, doi: 10.1029/2003JA010099, 2004.

Landau, L. D. and Lifshitz, E. M.: Electrodynamics of continuous media, vol. 8, Pergamon Press,, 1960.

Lepping, R. P. and Behannon, K. W.: Magnetic field directional discontinuities. I - Minimum variance errors, J. Geophys. Res., 85, 4695-4703, 1980.

Lepping, R. P. and Behannon, K. W.: Magnetic field directional discontinuities - Characteristics between 0.46 and 1.0 AU, J. Geophys. Res., 91, 8725-8741, 1986.
Lyons, L. R.: Substorms: Fundamental observational features, distinction from other disturbances, and external triggering, J. Geophys. Res., 101, 13 011-13 026, doi:10.1029/95JA01987, 1996.

Lyons, L. R., Liu, S., Ruohoniemi, J. M., Solovyev, S. I., and Samson, J. C.: Observations of dayside convection reduction leading to substorm onset, J. Geophys. Res. (Space Physics), 108, doi: 10.1029/2002JA009670, 2003.

McComas, D. J., Bame, S. J., Barker, P., Feldman, W. C., Phillips, J. L., Riley, P., and Griffee, J. W.: Solar Wind Electron Proton Alpha Monitor (SWEPAM) for the Advanced Composition Explorer, Space Sci. Rev., 86, 563-612, 1998.

Neugebauer, M.: Comment on the abundances of rotational and tangential discontinuities in the solar wind, J. Geophys. Res., 111, A04103, doi:10.1029/2005JA011497, 2006.

Neugebauer, M., Clay, D. R., Goldstein, B. E., Tsurutani, B. T., and Zwickl, R. D.: A reexamination of rotational and tangential discontinuities in the solar wind, J. Geophys. Res., 89, 53955408, 1984.

Ogino, T., Walker, R. J., and Ashour-Abdalla, M.: A global magnetohydrodynamic simulation of the response of the magnetosphere to a northward turning of the interplanetary magnetic field, J. Geophys. Res., 99, $11027-11042$, doi:10.1029/ 93JA03313, 1994.

Papitashvili, V. O. and Rich, F. J.: High-latitude ionospheric convection models derived from Defense Meteorological Satellite Program ion drift observations and parameterized by the interplanetary magnetic field strength and direction, J. Geophys. Res., 107, 17-1, doi:10.1029/2001JA000264, 2002.

Rème, H., Aoustin, C., Bosqued, J. M., Dandouras, I., Lavraud, B., Sauvaud, J. A., Barthe, A., Bouyssou, J., Camus, T., Coeur-Joly, O., Cros, A., Cuvilo, J., Ducay, F., Garbarowitz, Y., Medale, J. L., Penou, E., Perrier, H., Romefort, D., Rouzaud, J., Vallat, C., Alcaydé, D., Jacquey, C., Mazelle, C., D’Uston, C., Möbius, E., Kistler, L. M., Crocker, K., Granoff, M., Mouikis, C., Popecki, M., Vosbury, M., Klecker, B., Hovestadt, D., Kucharek, H., Kuenneth, E., Paschmann, G., Scholer, M., Sckopke, N., Seidenschwang, E., Carlson, C. W., Curtis, D. W., Ingraham, C., Lin, R. P., McFadden, J. P., Parks, G. K., Phan, T., Formisano, V., Amata, E., Bavassano-Cattaneo, M. B., Baldetti, P., Bruno, R., Chionchio, G., di Lellis, A., Marcucci, M. F., Pallocchia, G., Korth, A., Daly, P. W., Graeve, B., Rosenbauer, H., Vasyliunas, V., McCarthy, M., Wilber, M., Eliasson, L., Lundin, R., Olsen, S., Shelley, E. G., Fuselier, S., Ghielmetti, A. G., Lennartsson, W., Escoubet, C. P., Balsiger, H., Friedel, R., Cao, J.-B., Kovrazhkin, R. A., Papamastorakis, I., Pellat, R., Scudder, J., and Sonnerup, B.: First multispacecraft ion measurements in and near the Earth's magnetosphere with the identical Cluster ion spectrometry (CIS) experiment, Ann. Geophys., 19, 1303-1354, 2001 , http://www.ann-geophys.net/19/1303/2001/.

Ridley, A. J.: Estimations of the uncertainty in timing the relationship between magnetospheric and solar wind processes, J. Atmos. Solar-Terr. Phys., 62, 757-771, 2000.

Ruohoniemi, J. M. and Greenwald, R. A.: Dependencies of high-latitude plasma convection: Consideration of interplanetary magnetic field, seasonal, and universal time factors in statistical patterns, J. Geophys. Res., 110, A09204, doi:10.1029/ 2004JA010815, 2005.

Sergeev, V. A., Dmitrieva, N. P., and Barkova, E. S.: Triggering of 
substorm expansion by the IMF directional discontinuities: Time delay analysis, Planet. Space Sci., 34, 1109-1118, doi:10.1016/ 0032-0633(86)90023-1, 1986.

Siscoe, G. L., Davis, Jr., L., Coleman Jr., P. J., Smith, E. J., and Jones, D. E.: Power Spectra and Discontinuities of the Interplanetary Magnetic Field: Mariner 4, J. Geophys. Res., 73, 61-79, 1968.

Smith, C. W., L'Heureux, J., Ness, N. F., Acuña, M. H., Burlaga, L. F., and Scheifele, J.: The ACE Magnetic Fields Experiment, Space Sci. Rev., 86, 613-632, 1998.

Smith, E. J.: Identification of interplanetary tangential and rotational discontinuities., J. Geophys. Res., 78, 2054-2063, 1973.

Snekvik, K., Haaland, S., Østgaard, N., Hasegawa, H., Nakamura, R., Takada, T., Juusola, L., Amm, O., Pitout, F., Rème, H., Klecker, B., and Lucek, E. A.: Cluster observations of a field aligned current at the dawn flank of a bursty bulk flow, Ann. Geophys., 25, 1405-1415, 2007, http://www.ann-geophys.net/25/1405/2007/.

Sonnerup, B. U. Ö. and Cahill, L.: Magnetopause structure and altitude from Explorer-12 observations, J. Geophys. Res., 72, 171183, 1967.

Sonnerup, B. U. Ö. and Scheible, M.: Minimum and Maximum Variance Analysis, in: Analysis Methods for Multi-Spacecraft Data, edited by: Paschmann, G. and Daly, P. W., ISSI SR-001, p. 1850, ESA Publications Division, 1998.

Sonnerup, B. U. Ö., Haaland, S., Paschmann, G., Dunlop, M. W., Rème, H., and Balogh, A.: Orientation and motion of a plasma discontinuity from single-spacecraft measurements: Generic residue analysis of Cluster data, J. Geophys. Res., 111, 52035223, doi:10.1029/2005JA011538, 2006.

Sonnerup, B. U. Ö., Haaland, S., and Paschmann, G.: Discontinuity Orientation, Motion and Thickness, in: Multi-spacecraft Analysis Methods Revisited, edited by Paschmann, G. and Daly, P. W., ISSI SR-005, pp. 1-10, ESA Publications Division, 2008.

Tsurutani, B. T. and Ho, C. M.: A review of discontinuities and Alfvén waves in interplanetary space: Ulysses results., Rev. Geophys., 37, 517-541, 1999.

Tsurutani, B. T. and Smith, E. J.: Interplanetary discontinuities Temporal variations and the radial gradient from 1 to $8.5 \mathrm{AU}, \mathrm{J}$. Geophys. Res., 84, 2773-2787, 1979.
Tsurutani, B. T., Guarnieri, F. L., Lakhina, G. S., and Hada, T.: Rapid evolution of magnetic decreases (MDs) and discontinuities in the solar wind: ACE and Cluster, Geophys. Res. Lett., 32, 10 103, doi:10.1029/2004GL022151, 2005.

Tsyganenko, N. A.: A model of the near magnetosphere with a dawn-dusk asymmetry 1. Mathematical structure, J. Geophys. Res., 107, 12-1, doi:10.1029/2001JA000219, 2002a.

Tsyganenko, N. A.: A model of the near magnetosphere with a dawn-dusk asymmetry 2. Parameterization and fitting to observations, J. Geophys. Res., 107, 10-1, doi:10.1029/2001JA000220, 2002 b.

Turner, J. M. and Siscoe, G. L.: Orientations of "rotational" and "tangential" discontinuities in the solar wind., J. Geophys. Res., 76, 1816-1822, 1971.

Volwerk, M.: Multi-Satellite observations of ULF waves, in: Magnetospheric ULF waves: Synthesis and new directions, edited by: Takahashi, K., Chi, P. J., Denton, R. E., and Lysak, R. L., pp. 109-135, AGU, Washington, 2006.

Weimer, D. R.: Correction to "Predicting interplanetary magnetic field (IMF) propagation delay times using the minimum variance technique", J. Geophys. Res., 109, 12 104, doi:10.1029/ 2004JA010691, 2004.

Weimer, D. R.: Improved ionospheric electrodynamic models and application to calculating Joule heating rates, J. Geophys. Res., 110, 5306, doi:10.1029/2004JA010884, 2005.

Weimer, D. R. and King, J. H.: Improved calculations of IMF phase front angles and propagation time delays, J. Geophys. Res., 113, A01105, doi:10.1029/2007JA012452, 2008.

Weimer, D. R., Ober, D. M., Maynard, N. C., Burke, W. J., Collier, M. R., McComas, D. J., Ness, N. F., and Smith, C. W.: Variable time delays in the propagation of the interplanetary magnetic field, J. Geophys. Res., 107, 29-1, doi:10.1029/2001JA009102, 2002.

Weimer, D. R., Ober, D. M., Maynard, N. C., Collier, M. R., McComas, D. J., Ness, N. F., Smith, C. W., and Watermann, J.: Predicting interplanetary magnetic field (IMF) propagation delay times using the minimum variance technique, J. Geophys. Res., 108, 16-1, doi:10.1029/2002JA009405, 2003. 